\title{
Why Does the Brain Predict Sensory Consequences of Oculomotor Commands? Optimal Integration of the Predicted and the Actual Sensory Feedback
}

\author{
Siavash Vaziri, Jörn Diedrichsen, and Reza Shadmehr \\ Laboratory for Computational Motor Control, Department of Biomedical Engineering, Johns Hopkins University, Baltimore, Maryland 21205
}

\begin{abstract}
When the brain initiates a saccade, it uses a copy of the oculomotor commands to predict the visual consequences: for example, if one fixates a reach target, a peripheral saccade will produce an internal estimate of the new retinal location of the target, a process called remapping. In natural settings, the target likely remains visible after the saccade. So why should the brain predict the sensory consequence of the saccade when after its completion, the image of the target remains visible? We hypothesized that in the post-saccadic period, the brain integrates target position information from two sources: one based on remapping and another based on the peripheral view of the target. The integration of information from these two sources could produce a less variable target estimate than is possible from either source alone. Here, we show that reaching toward targets that were initially foveated and remapped had significantly less variance than reaches relying on peripheral target information. Furthermore, in a more natural setting where both sources of information were available simultaneously, variance of the reaches was further reduced as predicted by integration. This integration occurred in a statistically optimal manner, as demonstrated by the change in integration weights when we manipulated the uncertainty of the post-saccadic target estimate by varying exposure time. Therefore, the brain predicts the sensory consequences of motor commands because it integrates its prediction with the actual sensory information to produce an estimate of sensory space that is better than possible from either source alone.
\end{abstract}

Key words: forward models; efferent copy; optimal integration; reaching; Bayesian models; motor control

\section{Introduction}

Consider a situation in which a person is reading a book and decides to reach for a cup located in her peripheral vision. She saccades to the cup, then saccades back to the book, and after a short delay initiates the reach. Previous studies suggest that the position of the reach target (the cup) is coded in fixationcentered coordinates (Henriques et al., 1998; Batista et al., 1999; Pouget et al., 2002). Furthermore, near the time of the second saccade, this fixation-centered representation is updated: cells in many areas of the brain exhibit "remapping" (Duhamel et al., 1992; Walker et al., 1995; Umeno and Goldberg, 1997; Batista et al., 1999; Nakamura and Colby, 2002; Medendorp et al., 2003; Merriam et al., 2003). That is, cells with retinocentric receptive fields that will cover the cup after the saccade begin to discharge in anticipation of that visual input. However, as the saccade takes place, why would the brain predict the resulting location of the cup? In the natural setting, the actual image of the cup will appear

\footnotetext{
Received Nov. 4, 2005; revised March 5, 2006; accepted March 9, 2006.

This work was supported by National Institutes of Health Grant NS37422 and by the Human Frontiers Science Program. We thank Mark Shelhamer for helpful comments.

Correspondence should be addressed to Dr. Reza Shadmehr, Department of Biomedical Engineering, Johns Hopkins School of Medicine, 720 Rutland Avenue, 419 Traylor Building, Baltimore, MD 21205-2195. E-mail: reza@bme.jhu.edu.

DOI:10.1523/JNEUROSCI.4747-05.2006

Copyright $\odot 2006$ Society for Neuroscience $\quad 0270-6474 / 06 / 264188-10 \$ 15.00 / 0$
}

in peripheral vision shortly after completion of the saccade, making prediction seemingly superfluous.

One possibility is that the estimate of target location as sensed through peripheral vision is inaccurate; perhaps the mean is biased (Bock, 1986). Foveating the target and remapping that location might result in a removal of this bias. However, Henriques et al. (1998) and others (Medendorp and Crawford, 2002; Pouget et al., 2002; Poljac and Van Den Berg, 2003) demonstrated that reaches to remapped targets are biased in the same way as reaches to peripheral targets. In experiment 1 , we confirmed this result. However, we found that the remapped estimate of the target had better precision (smaller variance) than the estimate derived from peripheral vision.

In our example, the cup will not disappear after the person saccades back to the book. This implies that after completion of the saccade, the brain has two sources of information for estimating the location of the cup: one predicted through remapping and the other estimated from real-time peripheral vision. In light of recent work on the integration of two sensory perceptions (van Beers et al., 1999; Ernst and Banks, 2002) or on the integration of a sensory perception and a memory (Niemeier et al., 2003; Körding and Wolpert, 2004), we hypothesized that in the postsaccadic period, the brain might integrate the two sources of information to form a better estimate of target position. The optimal estimate would take into account the variance of both the predicted and the actual sensory information and weigh them by 
A

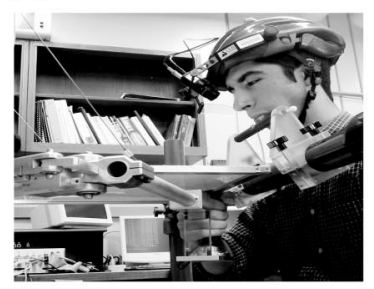

C

\section{Experiment 1}

Control

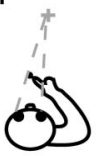

B
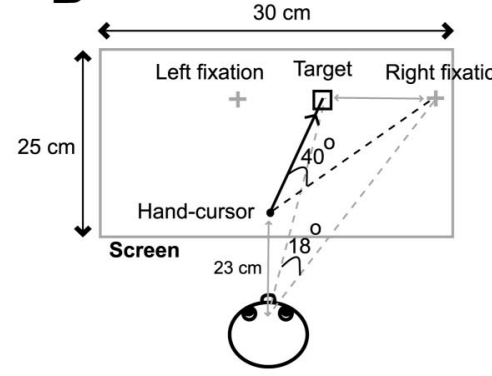

D

\section{Experiment 2}

Control

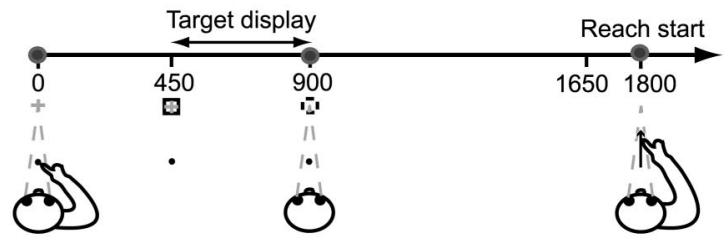

Target display:

Static
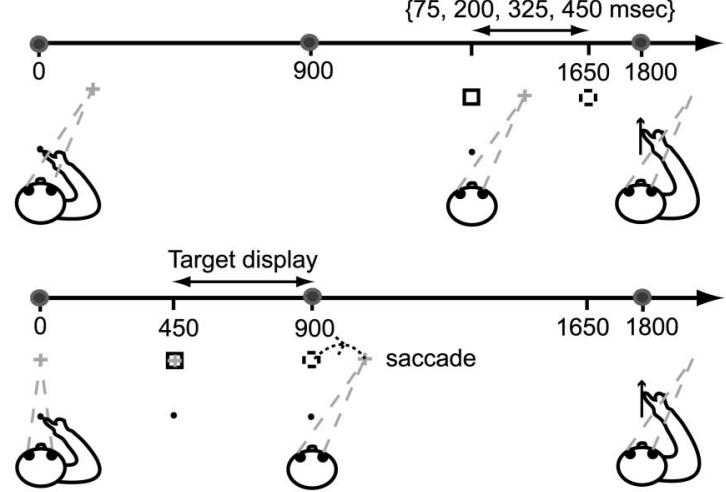

Remap

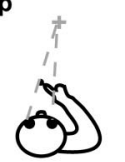

ㅁ

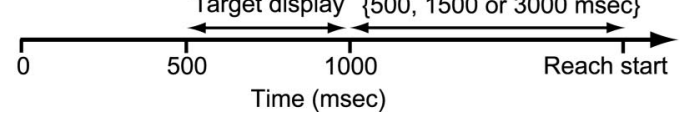

Remap
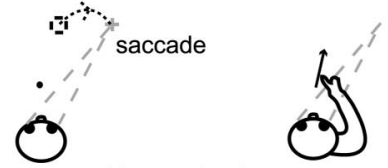

Memory load: Target display $\{500,1500$ or $3000 \mathrm{msec}\}$
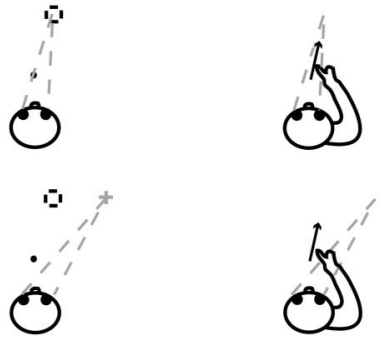

remap

Time (msec)

Figure 1. Experimental setup and conditions. $A$, Subjects used a robotic arm to make planar reaching movements to visual targets that were displayed on an opaque screen that was suspended above the robot handle. The reach target and other visual markers used in the experiment were displayed using an LCD projector held from the ceiling. Direct visual feedback of the hand and arm was prevented by covering the space around the screen using a dark cloth (data not shown). Eye position was measured with an infrared camera attached to a helmet, and a bite bar was used to stabilize the head. $\boldsymbol{B}$, The reach target, fixation point, and a cursor indicating robot position (beneath screen) were displayed on a $30 \times 25 \mathrm{~cm} \mathrm{screen.} \mathrm{The} \mathrm{hand} \mathrm{starting} \mathrm{position} \mathrm{was} 23 \mathrm{~cm}$ away from the subjects' cyclopean eye position. Angular position can either be expressed relative to the starting location of the hand (reach angle) or expressed relative to the cyclopean eye position (visual angle). C, Conditions in experiment 1 began with the display of a fixation cross (gray crosshair) and hand-cursor (small black circle). The dashed gray lines converging at the fixation point indicate the direction of gaze. The reach target (solid black square) was presented from 500 to $1000 \mathrm{~ms}$. The target was extinguished (dotted black square) at $1000 \mathrm{~ms}$ (as well as fixation cross in the control condition only), and a variable memory delay $(500,1500$, or $3000 \mathrm{~ms}$ in duration) followed in all conditions. In the remapping condition, the fixation cross jumped (black dotted arrow) at $1000 \mathrm{ms,}$ requiring a saccade to the new fixation position. At the end of the memory delay, an auditory tone instructed the subjects to initiate their reach toward the remembered target location. The fixation cross and hand-cursor disappeared before start of the reach. $\boldsymbol{D}$, Events in conditions of experiment 2 were arranged based on auditory tones delivered at 0,900 , and $1800 \mathrm{~ms}$ (gray circles on timelines). These tones corresponded to the following events: appearance of fixation cross, jump in fixation cross requiring saccade (remapping and combined only), and, finally, reach start. Reach target exposure times for each condition are labeled as "Target display" on the timelines. Variable exposure lengths $(75,200,325$, or $450 \mathrm{~ms})$ were used in the static condition and the second target display of the combined condition that could also appear at variable positions of $0,-5$, or $+5^{\circ}$ with respect to the first target display (pre-saccadic). In all conditions, visual markers (fixation cross and hand-cursor) still visible at $1650 \mathrm{~ms}$ were extinguished, signaling subjects to begin the reach at $1800 \mathrm{~ms}$ with the arrival of the final tone. Remap, Remapping.

the inverse of their variances. In experiment 2, we tested this prediction. We manipulated the uncertainty of peripheral information by varying the length of target exposure and tested whether the weights of the integration changed accordingly. We found that the brain combined the remapped target estimate with an estimate derived from peripheral vision, and this resulted in a more precise reach than was possible from each source of information alone.

\section{Materials and Methods}

As shown in Figure $1 \mathrm{~A}$, subjects were seated in front of a Plexiglas screen $(30 \times 25 \mathrm{~cm})$ that was suspended in the horizontal plane above a planar ( 2 degrees of freedom) robotic arm (Ariff et al., 2002; Nananyakkarra and Shadmehr, 2003). Background light was minimized; however, the edges of the screen were visible. The center of the screen was at a distance of 30 $\mathrm{cm}$ from the subjects' eyes (mid-point), measured while the head was stabilized with a bite bar (adjustable in vertical direction). As a result, the screen was approximately located below shoulder level. The screen was made opaque with thick white paper covering the viewable topside. This prevented the subject from seeing their hands below the screen. The subjects were asked to hold the robot handle and make reaching movements to targets that were displayed on the screen using a liquid crystal display (LCD) projector (attached to ceiling). The position of the robot handle was displayed on the screen using a cursor (Fig. $1 B$ ). Therefore, what is referred to as a reaching movement involved the relocation of the robot handle to bring the cursor to the target. Because the cursor viewed by the subjects on the screen was directly above the robot handle, the relationship between movements of the robot and corresponding changes in cursor position was very easy to learn. Visual feedback of the hand and arm was prevented by using a dark cloth draped around the screen. Therefore, the only visual feedback about hand position came from the hand-cursor. Apart from the pretraining session (details explained later), reaching movements were made to the remembered location of the target. Furthermore, before the movement began, the handcursor was turned off and thereby removed hand visual feedback during the movement. 
Measurement of hand position was obtained from the robot with a resolution of $0.05 \mathrm{~mm}$. Eye position was monitored using a headmounted infrared light source and camera (gaze accuracy of $1^{\circ}, 100 \mathrm{~Hz}$ sampling rate; iView system, SensoMotoric Instruments, Teltow, Germany). At the start of every set of movements (110 trials), the eye system was recalibrated by having the subject fixate 24 targets of known position on the screen.

Two experiments were performed on separate groups of subjects all naive to the purpose of the experiments. The Johns Hopkins University Institutional Review Board approved the experimental protocols, and all subjects signed a consent form.

Experiment 1. Ten right-handed subjects (five males and five females; mean age, 22.5 years) were used. They made reaching movements under three conditions (Fig. 1C): control, static, and remapping. All conditions began with the robot positioning the hand at the start position located at a point along the midline and the projector displaying a cursor (circle, $0.15 \mathrm{~cm}$ diameter) indicating current hand position. A fixation cross (crosshair, $0.8 \mathrm{~cm}$ in length and width) appeared and indicated the start of the trial. The position of the fixation cross depended on the condition associated with that trial, as explained later for each condition. After 500 $\mathrm{ms}$ of fixation, a reach target (square, $0.9 \mathrm{~cm}$ side length) was displayed for a duration of $500 \mathrm{~ms}$. The target appeared at a distance of $11 \mathrm{~cm}$ and a direction of either $+10^{\circ}$ or $-10^{\circ}$ (randomly selected) with respect to the starting hand position (the $+10^{\circ}$ target is shown in Fig. 1C). During target display, the fixation cross remained visible and subjects maintained fixation.

In the "control condition," the fixation cross appeared at the same position as the reach target. This allowed for the natural behavior of reaching to the fixation point.

In the "static condition," the fixation cross was displayed at an eccentricity of $\pm 18^{\circ}$ visual angle from the reach target. This corresponded to an angle of $\pm 40^{\circ}$ with respect to the hand starting position. Therefore, the static condition resulted in a peripheral viewing of the target.

In the "remapping condition," the fixation cross was initially shown at the same position as the reach target. After the end of target display, the fixation cross jumped $+18^{\circ}$ or $-18^{\circ}$, resulting in a saccade and a remapping of the remembered target position. Therefore, the remapped position of the reach target had the same eccentricity as the target in the static condition.

In all conditions, a variable memory delay of 500, 1500, or $3000 \mathrm{~ms}$ started after the reach target was extinguished at $1000 \mathrm{~ms}$ into the trial. Note that the fixation cross was also extinguished at $1000 \mathrm{~ms}$ for the control condition, whereas it remained visible for the static and remapping conditions. At the end of the memory delay, both the hand-cursor and fixation cross (for remapping and static) disappeared and an auditory cue signaled the subject to reach to the remembered location of the target. Subjects were instructed to maintain gaze direction until the movement had ended. The movements had to start within $250 \mathrm{~ms}$ after the auditory cue and end within 460-550 ms. If these constraints were satisfied, a pleasing sound was generated. Otherwise, the subjects received the following feedback about failure to meet the constraints: (1) the words "late" or "early" were displayed to give feedback concerning the timing of reach start; or (2) the words "slow" or "fast" were displayed to give feedback regarding the duration of the reach. The trial concluded with the robot bringing the hand back to the start position. Trials with visual feedback of the hand (hand-cursor on) were presented occasionally ( 1 in 10 trials) to prevent degradation of movement accuracy caused by proprioceptive drift (Wann and Ibrahim, 1992). Reaching, however, was still made to the memorized location of the target to prevent visual error feedback. These trials were excluded from the analysis.

There were 15 possible combinations of condition, delay, and fixation position. Each combination was repeated 20 times for a total of 300 trials, divided into three sets. The combinations were interleaved randomly.

Before the start of the main experiment, the subjects participated in two sessions of training. During session 1, we provided complete visual feedback of the hand (hand-cursor on) and displayed the reach target throughout the trial. In these trials, reaching movements were performed without any constraints on gaze position. This training session allowed the subjects to learn the mapping between changes in robot and cursor position (learning occurred in 5-10 trials). During session 2, we provided the actual conditions of the experiment until the subjects became comfortable with the gaze requirements of each condition (usually $\sim 100$ trials). Note that during these trials, movements were still made to the memorized target position and therefore the subjects never received visual error feedback. We tested for the effect of training by looking for changes in movement error as a function of set number. Training could possibly lead to an improvement in performance; however, we did not find any significant changes in performance (bias and variance) across sets.

Experiment 2. Five right-handed subjects (four males and one female; mean age, 27.6 years) were used. They were tested in conditions designed to investigate whether remapped and static target estimates are combined when both are available before reaching (Fig. $1 D$ ). A trial began with the robot positioning the hand at the midline start position and the computer selecting the position of the upcoming reach target (the target was not displayed yet). The selected target position was chosen from a sequence of targets having directions in the range of -10 to $+10^{\circ}$ (generated from a uniform distribution) with respect to the starting hand position (the $0^{\circ}$ is shown in Fig. $1 D$ ). The distance of the selected target from the start position was $11 \mathrm{~cm}$. After the robot brought the hand to the start position, the hand-cursor and the fixation cross were displayed to mark the start of the trial.

We attempted to tightly control when participants initiated their saccades and reaches. To achieve this, auditory tones were delivered at 0 , 900 , and $1800 \mathrm{~ms}$ into the trial. The first tone signaled the subject to fixate the fixation target. The second tone corresponded to the time when the fixation crosshair jumped to the periphery, requiring a saccade in the remapping and combined conditions. The final tone at $1800 \mathrm{~ms}$ signaled the subject to begin the reach.

In the control condition, the fixation cross was shown at the position of the upcoming reach target, signaling the start of the trial. At $450 \mathrm{~ms}$, the reach target was displayed. At $900 \mathrm{~ms}$, both the fixation cross and the reach target disappeared. However, subjects continued to maintain their gaze. The hand-cursor disappeared at $1650 \mathrm{~ms}$ into the trial, signaling subjects to prepare for the reach. The reach was triggered at $1800 \mathrm{~ms}$ with the presentation of the last auditory tone.

In the static condition, the fixation cross was displayed at $\pm 18^{\circ}$ with respect to the target location. At 1200, 1325, 1450, or $1575 \mathrm{~ms}$, the target was displayed while the subject continued to maintain fixation. At 1650 $\mathrm{ms}$, the reach target, the fixation cross, and the hand-cursor disappeared. Therefore, the duration of target exposure was $75,200,325$, or $450 \mathrm{~ms}$. The reach was triggered at $1800 \mathrm{~ms}$ with the presentation of the last auditory tone.

In the remapping condition, an initial fixation cross was displayed at the position of the upcoming target. The target was displayed at $450 \mathrm{~ms}$. At $900 \mathrm{~ms}$, the target disappeared and the fixation cross jumped $\pm 18^{\circ}$ with respect to the target direction, instructing a saccade. At $1650 \mathrm{~ms}$, the reach target, the fixation cross, and the hand-cursor disappeared. The reach was triggered at $1800 \mathrm{~ms}$ with the presentation of the last auditory tone.

In the "combined condition," the events were the same as in the remapping condition up until $1200 \mathrm{~ms}$ into the trial. After the saccade, the target then reappeared at $1200,1325,1450$, or $1575 \mathrm{~ms}$ as in the static condition. The second target presentation was at either $0^{\circ}$ (unshifted) or shifted by a visual angle of $\pm 2^{\circ}$ (shifted) with respect to the first reach target position (Fig. $1 D$, three black squares). The $\pm 2^{\circ}$ shift in the second target presentation corresponded to a change of $\pm 5^{\circ}$ in the reach angle.

The use of auditory tones as cues for controlling the timing of saccades was important because in the combined condition, we wanted the subjects to complete their saccade before the second target presentation began. In this way, the second target exposure would be completely peripheral as desired. In the combined condition (exposure, $450 \mathrm{~ms}$ ), subjects only had $300 \mathrm{~ms}$ to complete their saccade before the second target was displayed. The average saccade reaction time to the change in fixation position was $153 \mathrm{~ms}$ (SD, $54 \mathrm{~ms}$ ). Therefore, by using auditory tones, we were able to significantly reduce the number of trials excluded from analysis because of delayed saccades. Also, it was important to control the timing of reaches given the strong dependence of the variability of 


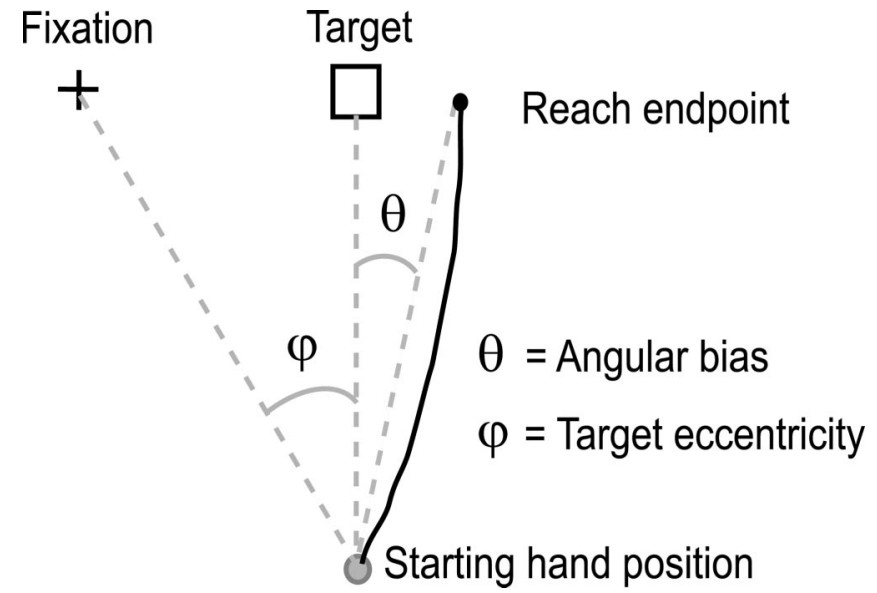

Figure 2. Performance measure. The error in the reaching movement (solid black line) was quantified by the angle $(\theta)$ between the movement and target direction. Movement direction was defined by a line (gray dashed) connecting the start position (gray circle) and the end point of the reach (black dot). The angle $(\varphi)$ between the fixation cross (black cross) and target with respect to the starting hand position was used as a measure of target eccentricity. The equivalent measure of target eccentricity in visual angles is also reported in the Results.

reaches on the memory interval found in experiment 1 . Reach reaction times (255 ms; SD, $79 \mathrm{~ms}$ ) were not significantly different across conditions.

We used the same performance feedback scheme as in experiment 1. Additionally, we gave a movement accuracy score to motivate the subjects to reach accurately: trials were binned into groups of 10 , and the average distance of the reach end points with respect to the target was displayed after at every 10th trial.

There were a total of 35 different combinations of conditions, fixations, exposure levels, and target shifts: control (1), remapping (2 fixations), static ( 2 fixations $\times 4$ exposures), combined ( 2 fixations $\times 4$ exposures $\times 3$ positions for the second target). Each combination was repeated 32 times during the experiment. Because of a large number of trials, the experiment was broken down into four sessions and each session was performed on a separate day. A session consisted of four sets of 70 trials with brief rest periods between each set. Within each set, every 10th trial was a control visual feedback trial similar to the first experiment. The initial training protocol was identical to that described for experiment 1 . In addition, at the beginning of each session, participants performed a set of reaching movements under complete visual feedback and a constantly lit target to refamiliarize them with the robotic arm. Again, as described for experiment 1, we did not find a change in movement performance (bias and variance) across sets. This indicates that training did not influence movement errors.

The static condition in either experiment used targets at an eccentricity of $40^{\circ}$ (visual angle, $18^{\circ}$ ). To investigate the effect of target eccentricity on reach errors, we performed an additional experiment $(n=7)$ in which participants performed reaching movements in a static condition (identical to experiment 1 ) with target eccentricities at 20,40 , or $60^{\circ}$ (visual angles: 8,18 , or $32^{\circ}$ ).

Performance measure. We connected the start and the end point of each reach and measured the angular deviation with respect to a straight line to the target (Fig. 2). The sign of the resulting angle depended on whether the reach was directed toward or away from the fixation. For example, missing the target $2^{\circ}$ (with respect to the starting location of the hand) to the right during leftward fixation was considered an error of $+2^{\circ}$. The same reach under right fixation was assigned a value of $-2^{\circ}$ (underestimation). By using coding relative to fixation, we were able to combine the reach errors for the left and right fixation. In this way, for both fixation directions, positive angular errors indicated overestimation of target eccentricity, whereas negative errors indicated underestimation of target eccentricity. The bias in the control condition (i.e., the condition in which gaze was on target) served as a baseline measure of error for each subject. We subtracted this baseline bias from the reaching error that we measured when gaze was not on target (static, remapping, and combined conditions). Errors in depth were also quantified. However, we did not find any significant difference across conditions.

Integration model. In experiment 2, we hypothesized that the target estimate in the combined condition was computed as a weighted average of target estimates based on the remapped and post-saccadic target information. These constituent remapped and post-saccadic target estimates are each characterized, respectively, in the remapping and static conditions. Our characterization of a target estimate consists of its bias and variability that we approximated by the mean and variance of reaching errors made to that target. The weighted average model states that the target estimate in the combined condition, $x_{c}$, is a weighted average of the target estimate in the remapped condition, $x_{\mathrm{r}}$, and in the static condition, $x_{\mathrm{s}}$, as follows:

$$
x_{\mathrm{c}}=\alpha x_{\mathrm{r}}+(1-\alpha) x_{\mathrm{s}} .
$$

The parameter $\alpha$ determines the weight given to the target estimate in the remapped and static condition, respectively, and takes on values in the range $[0,1]$. Integration takes place when the value of $\alpha$ is $>0$ and $<1$. In contrast, a value of zero or unity indicates a target estimate in the combined condition that is equivalent to the estimate in either the static or remapped conditions and hence implies an absence of integration. To estimate $\alpha$, we applied the expected value and variance operators to both sides of equation 1 :

$$
\begin{gathered}
E\left[x_{\mathrm{c}}\right]=\alpha E\left[x_{\mathrm{r}}\right]+(1-\alpha) E\left[x_{\mathrm{s}}\right] \\
\operatorname{var}\left[x_{\mathrm{c}}\right]=\alpha^{2} \operatorname{var}\left[x_{\mathrm{r}}\right]+(1-\alpha)^{2} \operatorname{var}\left[x_{\mathrm{s}}\right] .
\end{gathered}
$$

Although parameter $\alpha$ could be calculated from either Equation 2 or Equation 3, the variance model, unlike the mean response model, is a quadratic function of $\alpha$ and therefore produces two solutions. Although the solution outside the range $[0,1]$ can be ignored, there will still be instances depending on the variances in which two valid solutions exist. To avoid the problem of multiple solutions, we solved for $\alpha$ using Equation 2:

$$
\alpha=\frac{E\left[x_{\mathrm{c}}\right]-E\left[x_{\mathrm{s}}\right]}{E\left[x_{\mathrm{r}}\right]-E\left[x_{\mathrm{s}}\right]} .
$$

It is clear from the denominator of Equation 4 that a difference in value between the remapped target estimate $E\left[x_{\mathrm{r}}\right]$ and the post-saccadic target estimate $E\left[x_{s}\right]$ is a prerequisite for calculating the integration weight $\alpha$. To establish this difference, we introduced a manipulation in the combined condition whereby a small shift was applied between the presaccadic and post-saccadic target presentation.

In experiment 2, we sought to investigate whether integration, if present, was optimal in minimizing the variance of the combined condition. This can be verified by comparing the actual weights obtained using Equation 4 with the predicted optimal weights. The weights that minimize the variance of the combined estimate are proportional to the inverse of the uncertainty (variance) of the constituent sources:

$$
\alpha_{\text {Optimal }}=\frac{\frac{1}{\operatorname{var}\left[x_{\mathrm{r}}\right]}}{\frac{1}{\operatorname{var}\left[x_{\mathrm{s}}\right]}+\frac{1}{\operatorname{var}\left[x_{\mathrm{r}}\right]}}=\frac{\operatorname{var}\left[x_{\mathrm{s}}\right]}{\operatorname{var}\left[x_{\mathrm{s}}\right]+\operatorname{var}\left[x_{\mathrm{r}}\right]} .
$$

This weight is, in fact, the solution of the minimization of $\operatorname{var}\left[x_{\mathrm{c}}\right]$ (Eq. 3) with respect to $\alpha$. Therefore, by plugging $\alpha_{\text {Optimal }}$ into Equation 3, we obtain the optimal $\operatorname{var}\left[x_{\mathrm{c}}\right]$ :

$$
\operatorname{var}\left[x_{\mathrm{c}}\right]_{\text {Optimal }}=\frac{\operatorname{var}\left[x_{\mathrm{r}}\right] \operatorname{var}\left[x_{\mathrm{s}}\right]}{\operatorname{var}\left[x_{\mathrm{s}}\right]+\operatorname{var}\left[x_{\mathrm{r}}\right]} .
$$

An alternative integration mechanism is one that is based on fixed weights. In this type of integration, the weights assigned to each estimate are constant and therefore independent of the uncertainty in the estimate. To differentiate between a fixed or optimal weighting mechanism, 
we varied the post-saccadic target exposure lengths to induce different levels of uncertainty. If integration is optimal, the weights should vary as a function of exposure levels, whereas fixed weights should remain unaffected.

We could not use Equations 5 and 6 directly because variance in a reaching movement is likely to be influenced by both the estimate of the target location and the motor noise associated with its execution (van Beers et al., 2004). If we label the reach directions in the combined, remapped, and static conditions with the random variables $r_{\mathrm{c}}, r_{\mathrm{r}}$, and $r_{\mathrm{s}}$ and assume that motor noise is independent of the noise associated target location estimate, we have the following:

$$
\begin{aligned}
& \operatorname{var}\left[r_{\mathrm{r}}\right]=\sigma_{\text {motor }}^{2}+\operatorname{var}\left[r_{\mathrm{r}}\right] \\
& \operatorname{var}\left[r_{\mathrm{s}}\right]=\sigma_{\text {motor }}^{2}+\operatorname{var}\left[r_{\mathrm{s}}\right] \\
& \operatorname{var}\left[r_{\mathrm{c}}\right]=\sigma_{\text {motor }}^{2}+\operatorname{var}\left[r_{\mathrm{c}}\right],
\end{aligned}
$$

where $\sigma_{\text {motor }}^{2}$ represents the variability attributable to motor noise. The implication of introducing motor noise into the model is that the measured variance in each reaching condition cannot be used directly to approximate the target estimate variability. Rather, we need some way to estimate the variance induced by motor noise and subtract it from the measured variance in the reaching conditions. We therefore introduced the control condition in which the target was fixated and assumed that the variance in the reaches made in this condition is a good estimate of the motor noise. We then used Equation 7 to arrive at variances of target positions.

\section{Results}

\section{Experiment 1: remapping reduced reach variance}

The average reach trajectories (mean movement time, $480 \pm 43$ $\mathrm{ms}$ ) for a typical subject in experiment 1 are shown in Figure $3 A$. The corresponding reach end points are shown in Figure $3 B$. When the target appeared in peripheral vision (static condition), either in the left or in the right visual field, the subject overestimated its eccentricity and therefore exhibited a positive bias. The same reaching bias was found in the remapping condition. However, the end point variability in the remapping condition at $0.5 \mathrm{~s}$ is smaller than in the static condition (Fig. $3 B$ ). In the control condition (fixation on target), the subject reached more accurately and with greater precision to the target; however, a small bias was still observed. The average reach direction in the control condition was used as a baseline when comparing remapping and static conditions across subjects. We performed a repeatedmeasures ANOVA with the factors fixation (left vs right), condition (static vs remapping), and delay $(0.5,1.5$, and $3 \mathrm{~s})$. Because we did not find a significant effect for fixation $\left(F_{(1,9)}=0.72 ; p=\right.$ 0.42 ), nor a significant interaction (condition by fixation: $F_{(1,9)}=$ $1.23, p=0.30$; delay by fixation: $\left.F_{(2,18)}=0.15, p=0.86\right)$, we combined the results for the left and right fixations. We observed that subjects overestimated the eccentricity of the target in both the static and remapping conditions (Fig. $4 A$ ) and that the bias in these two conditions was statistically equivalent $\left(F_{(1,9)}=0.85\right.$; $p=0.38)$. In addition, changes in the delay period had no significant effects on the patterns of bias (main effect of delay: $F_{(2,18)}=$ $1.1, p=0.35$; delay by condition interaction: $F_{(2,18)}=0.9, p=$ $0.43)$.

In contrast, the variability of the reach direction was signifi-
B
Control ( $0.5 \mathrm{sec}$ delay)

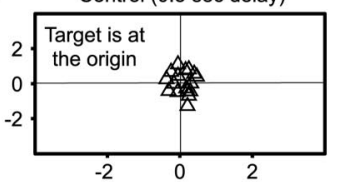

Static ( 0.5 sec delay)

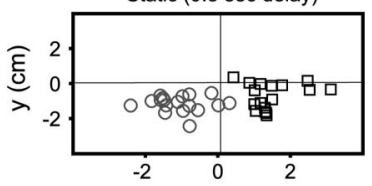

Remap (0.5 sec delay)

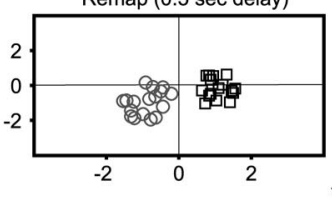

Control ( 3 sec delay)

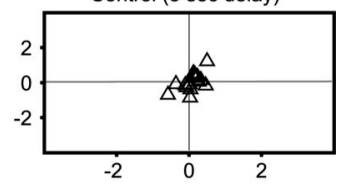

Static (3 sec delay)

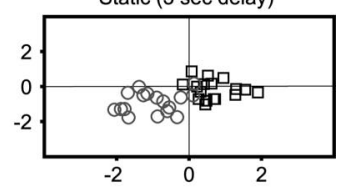

Remap (3 sec delay)

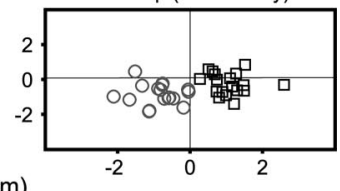

$x(\mathrm{~cm})$
A

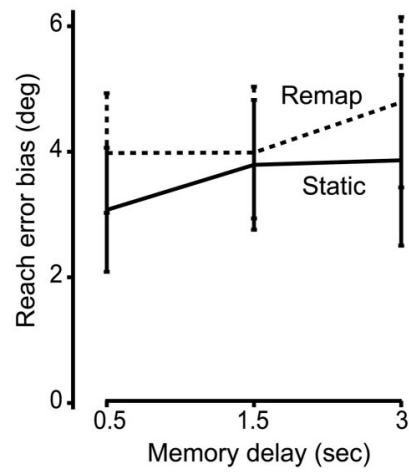

B

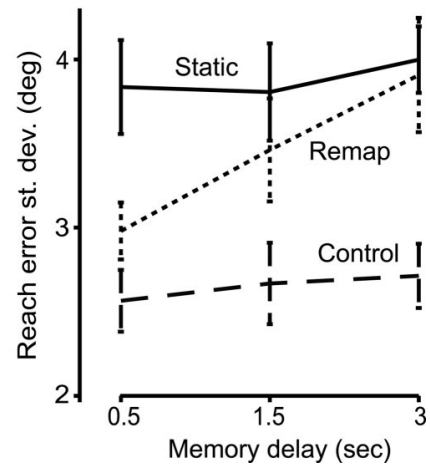

Figure 4. Average results of experiment 1. A, Bias of the reach error averaged over fixation (left and right) and subjects. Positive values indicate a bias away from the fixation point. The bias in the control condition for each subject was used as a baseline. $B, S D$ of reach errors as a function of condition and memory interval. Remap, Remapping.

cantly affected by the remapping and the passage of time (Fig. $4 B)$. A two-way, repeated-measures ANOVA with the factors condition (control, static, and remapping) and delay $(0.5,1.5$, and $3 \mathrm{~s})$ revealed a significant main effect of condition $\left(F_{(2,18)}=\right.$ $35.48 ; p \sim 0)$, delay $\left(F_{(2,18)}=4.60 ; p=0.024\right)$, and conditionby-delay interaction $\left(F_{(4,36)}=3.35 ; p=0.020\right)$. The data suggested that the effect of memory delay on variability is specific to the remapping condition (Fig. $4 B$ ). To test this idea, we performed a one-way, repeated-measures ANOVA for each condition separately and tested the main effect of delay. The effect of delay was only significant in the remapping condition $\left(F_{(2,18)}=\right.$ $8.51 ; p=0.003)$, but not in the control $\left(F_{(2,18)}=0.35 ; p=0.71\right)$ and static $\left(F_{(2,18)}=0.628 ; p=0.545\right)$ conditions. We found that the variability in the remapping condition increased from a value similar to the control condition at $0.5 \mathrm{~s}$ (remapping and control at $\left.0.5 s ; t_{(9)}=2.32 ; p=0.045\right)$ and approached the static variability at the longer memory delay of $3.0 \mathrm{~s}$ (remapping and static at $3 \mathrm{~s}$; $\left.t_{(9)}=0.32 ; p=0.76\right)$. Importantly, we found a highly significant improvement in the remapping variability over the static condition at a delay of $0.5 \mathrm{~s}\left(t_{(9)}=5.32 ; p<0.001\right)$. 
A

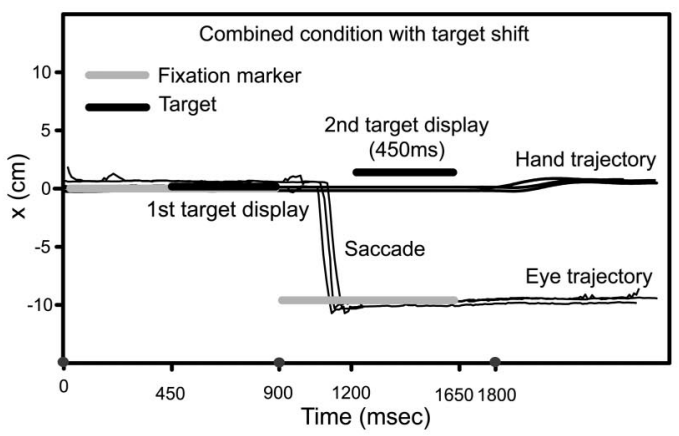

B

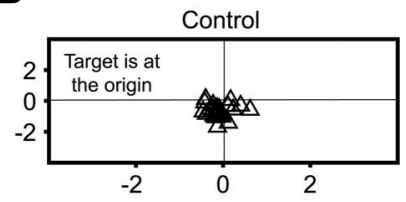

Static (75 ms exposure)

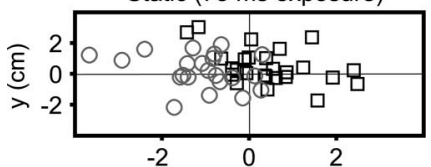

Combined (75 ms exposure)

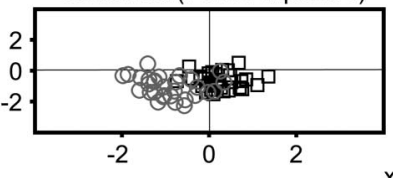

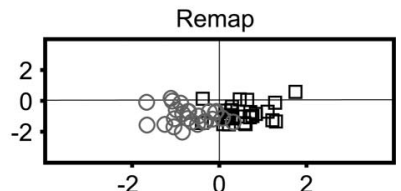

Static (450 ms exposure)

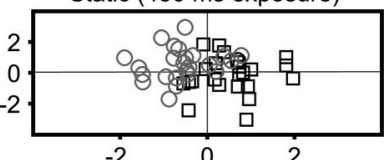

Combined (450 ms exposure)

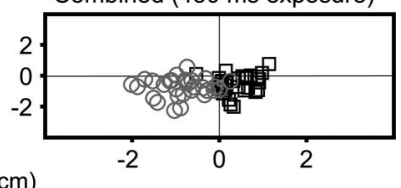

Figure 5. Single-subject reach performance in experiment 2. A, Sample eye and hand trajectories of the combined condition (target shift) in the $x$-direction. Gray circles on the time axis indicate when auditory tones were given. $\boldsymbol{B}$, Reach end points relative to the target (origin) in the control, remapping (Remap), static, and combined conditions. The end points for both the left (square) and the right (circle) fixation conditions and the 75 and 450 ms exposure levels are shown.

In summary, reaches that were performed after the target was remapped peripherally were equally biased as reaches performed after static viewing of the target in the periphery. However, at short memory intervals after the remapping, the remapped target estimate exhibited a smaller variance than the static condition target estimate. With larger memory delays, the variance associated with the remapped target increased.

\section{Experiment 2: the remapped target estimate was combined} with post-saccadic information to reduce reach variance

In the combined condition, participants initially fixated the reach target for $450 \mathrm{~ms}$. After making a saccade to the periphery, they were shown (for a variable exposure period) either the same or a slightly shifted version of the pre-saccadic target (Fig. 5A). Did the brain combine the visual information available after the saccade with the remapped visual information from before the saccade? If so, we can make the following predictions regarding the mean and variance of the reach trials in the combined condition:

(1) If the pre-saccadic and post-saccadic target information are integrated to improve the estimate of the target location, the reach variability for the combined condition should be smaller than the variability of both the static and remapping conditions. Furthermore, a lower bound for the variability of the combined condition is given by the values predicted by optimal integration (Eq. 6).

A

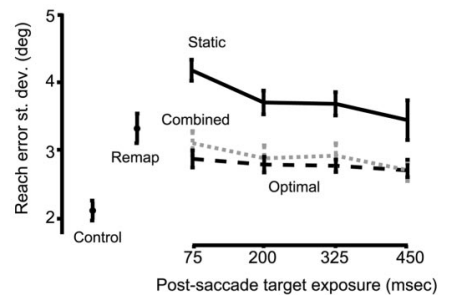

B

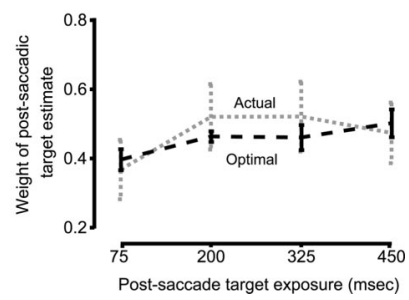

Figure 6. Average reach variance in experiment 2. $A, S D$ (st. dev.) in the control, remapping (Remap), static (solid black line; different target exposures), and combined (dotted gray line; different post-saccadic target exposures under no target shift) conditions averaged across fixations and subjects. The mean optimal variance (dashed black line) was calculated individually for each subject based on their static and remapping variability. Motor noise (control condition) was first subtracted from the individual variances (static and remapping) and then added back to the resulting optimal estimate calculated by Equation $6 . \boldsymbol{B}$, Average optimal weight of the static estimate (dashed black line) calculated (Eq. 5) separately for each subject based on the variability data in $\boldsymbol{A}$ (static and remapping conditions). We also show the actual weight (dotted gray line) found by fitting movement direction data of Figure 7 to Equation 4. These weights were calculated separately for the shift-away and shift-toward trials of the combined condition, and their averages are reported.

(2) When the post-saccadic target appears in a different location than the pre-saccadic target, integration predicts that the mean direction of the resulting reach should fall between two variables: the remapped estimate of the target and the estimate derived from the post-saccadic view of the target. If the integration is optimized, then the weight of each of these two variables should depend on the relative variance of the two sources of information (Eq. 5).

We first consider the variability of the reaches. Figure $5 B$ shows the reach end points from a single participant in the control, remapping, static, and combined (no target shift) conditions. The variability of the static condition at $75 \mathrm{~ms}$ is large, whereas the variability of the combined condition at the same target exposure level is markedly reduced. Figure $6 \mathrm{~A}$ shows the $\mathrm{SD}$ of the static, remapping, and combined conditions (only the trials without target shifts were included in this plot) averaged across fixations and subjects. We found that the variability was lowest in the control condition in which subjects fixated the reach target. The reach variability was largest in the static condition when the target exposure time was smallest $(75 \mathrm{~ms})$. As the length of target exposure increased in the static condition, the reach variability decreased $\left(F_{(3,12)}=3.95 ; p=0.029\right)$. The remapping variability was smaller than the static variability at the lowest target exposure of $75 \mathrm{~ms}$ (paired $t$ test; $t_{(4)}=4.33 ; p=0.007$ ). However, at $450 \mathrm{~ms}$, which equals the target exposure used in the remapping condition, the variability of the remapping and static conditions became similar. This is consistent with the result of experiment 1 (Fig. $4 B$ ): the memory delay of the remapping condition in experiment 2 was set to $900 \mathrm{~ms}$, and interpolation at this memory load using Figure $4 B$ predicts similar variability for the remapping and static conditions.

To test our first prediction, we compared the variability of the combined condition (Fig. 6A, dotted gray line) with the static condition and found it to be significantly smaller at all exposure periods (all $p<0.01$ ). The variability in the combined condition was also smaller compared with the remapping condition for all exposures, except $75 \mathrm{~ms}$ (all $p<0.05$ ). This suggested that the brain combined the two sources of information (remapped and post-saccadic) in a way that resulted in a target estimate that was better than the individual remapped and post-saccadic target es- 
timates. If the weighting of each source of information was optimal, then the resulting reach variance should be minimized. We therefore set out to calculate the theoretical lower bounds on reach variance in the combined condition. We used the variance in the control condition to represent motor noise and used Equation 7 to compute $\operatorname{var}\left[x_{\mathrm{r}}\right]$ and $\operatorname{var}\left[x_{\mathrm{s}}\right]$ (i.e., variance of the remapped and post-saccadic target estimates) from the measured variances in the corresponding reach condition (remapping and static). Substituting these values in Equation 5 produced the theoretically optimal weighting of the post-saccadic and remapped estimates of target position (Fig. $6 \mathrm{~B}$, dashed black line, Optimal). We then inserted the optimal weights in Equation 3 and computed the lower bounds for the variance of reach trials in the combined condition (Fig. 6A, , dashed black line, Optimal). Remarkably, we found that the actual variability of the reach trials in the combined condition was quite similar to the lower bounds predicted by an optimal integration model ( $p>0.1$ for 75,200 , and $450 \mathrm{~ms} ; p>0.05$ for $325 \mathrm{~ms}$ ).

To test our second prediction, we considered the mean reach direction in the condition in which after the saccade, a target appeared for a variable time at $\pm 2^{\circ}$ visual angle with respect to the pre-saccadic target (equal to a $5^{\circ}$ change in reach angle). We imagined that the resulting reach could be explained by one of three scenarios. In the first scenario, the reach plan relied entirely on the remapped target estimate and ignored the post-saccadic target exposure. In the second scenario, the reach plan relied entirely on the target estimate provided by the view of the postsaccadic target. In the third scenario, the reach plan had access to both target estimates (remapped and post-saccadic) and weighted the two as a function of their uncertainty.

The predictions of the first and second scenario are illustrated in Figure $7 \mathrm{~A}$. The line at $0^{\circ}$ corresponds to the pre-saccadic target position. The first column shows the remapping condition in which the target was remapped when participants made a saccade. As found in experiment 1 , remapping led to an overestimation of the distance of the target from the fixation point and resulted in biased reaching movements. We would predict similar reaching movements for the combined condition based on the first scenario in which the reach plan only relies only on the remapped target estimate and ignores post-saccadic information. The mean reach direction of the combined condition should be similar to the remapping condition regardless of the postsaccadic target exposure level (Fig. $7 B$, solid black line).

The second column in Figure $7 A$ shows the bias for the static condition at all exposure levels (solid black line, actual reach). At an exposure level of $450 \mathrm{~ms}$ (matching exposure for remapping), the bias is nearly identical to the remapping bias $\left(t_{(4)}=0.12 ; p=\right.$ 0.91 ) consistent with the results of experiment 1 . In the static phase of the combined condition, the post-saccadic target was presented at a shifted position of $\pm 5^{\circ}$ reach angle (Fig. $7 \mathrm{~A}$, dotted squares) with respect to the pre-saccadic target. If only the postsaccadic information is used in forming the reach plan (second scenario), then the reach should be equally biased for the new post-saccadic target. Therefore, the predicted mean reach direction under scenario 2 (black dotted lines in Fig. $7 \mathrm{~A}$ and replotted in Fig. $7 B$ ) is a shifted version of the mean reach direction associated with the static condition (solid black line) when the postsaccadic target shifts by $\pm 5^{\circ}$. This predication relies on the assumption that the reaching bias in the static condition based on a target eccentricity of $40^{\circ}$ (visual angle, $18^{\circ}$ ) resulted in a similar bias for targets at $35^{\circ}$ or $45^{\circ}$ (post-saccadic eccentricities of the combined condition at visual angles of 16 and $20^{\circ}$ ). To test this assumption, we ran a control study (see Materials and Methods).
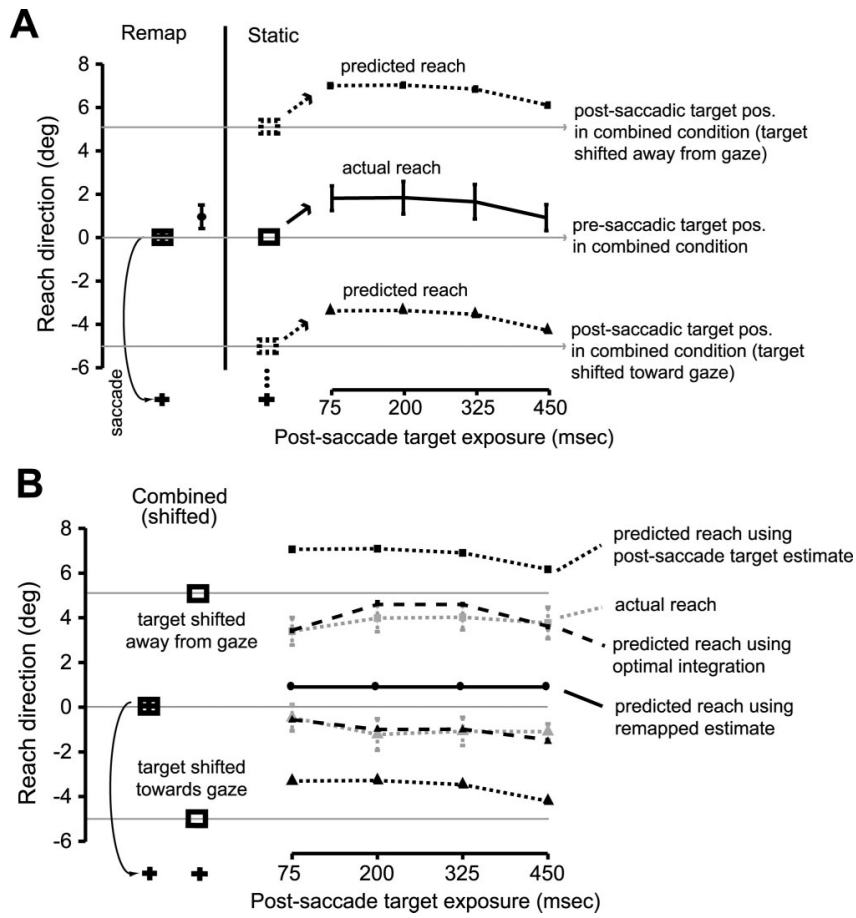

Figure 7. Average reach direction in experiment 2. A, Mean angular reach direction averaged (over fixation and subject) for the remapping (Remap; black circle) and static (solid black line for all exposures) conditions. The predicted reach direction in the combined condition in which target estimate is based on post-saccadic target exposure [dotted black line both for a post-saccadic target shifted away (square) and shifted toward gaze (triangle)] is also shown. All reach directions are plotted with respect to the pre-saccadic target position of the combined condition $\left(0^{\circ}\right.$ grid line). Reach directions are assigned a positive sign if directed away from gaze and a negative sign if directed toward gaze. $\boldsymbol{B}$, Mean angular reach direction of the combined condition [dotted gray line both for a post-saccadic target shifted away (square) and shifted toward gaze (triangle)] and the predicted optimal reach direction (dashed black line for each shift type). Measures were averaged over subjects and fixations.

Participants performed reaching movements in a static condition with target eccentricities at 20,40 , or $60^{\circ}$ (visual angles: 8,18 , or $32^{\circ}$ ). We found a small increase in bias from 20 to $40^{\circ}$ but no significant difference between 40 and $60^{\circ}$. Therefore, the reaching bias within the small range of $35-45^{\circ}\left(16-20^{\circ}\right.$ in visual angles) remained constant.

In the final scenario, the reach target is estimated as a weighted combination of the remapped and the post-saccadic target estimate. This predicts that the actual reach direction should fall somewhere between the prediction of the first two scenarios. The observed mean reach directions in the combined condition (Fig. $7 B$, dotted gray lines for each shift) supported this hypothesis. For a target shift in either direction, the movements were directed toward a location between the predicted reach using a remapped target estimate (Fig. $7 B$, solid black line) and the predicted reach using the post-saccade target estimate (Fig. $7 B$, dotted black line for each shift). Paired $t$ tests between the remappingand combined conditions revealed significant differences for all exposure levels and both shift directions (all $p<0.01$ ). Similar paired $t$ tests between the static and combined conditions at each exposure level and shift direction were also significant (all $p<0.05)$.

Because the measured reach variance was near the lower bounds predicted by optimal integration (Fig. $6 \mathrm{~A}$ ), the optimal weights should also be able to predict the mean direction of the reaching movements for the various exposure times. We inserted the weights plotted in Figure $6 B$ (dashed line) into Equation 2 
and plotted the resulting predicted mean reach direction for the $+5^{\circ}$ and $-5^{\circ}$ target shifts (Fig. $7 B$, dashed black lines). Remarkably, the actually measured mean reach direction in the combined condition (Fig. $7 B$, dotted gray lines) matched those predicted by optimal integration. To summarize, the measured biases of the reaching movements in the combined condition at various exposure times suggested that the reach plan was formed through an integration of the remapped estimate with the estimate derived from post-saccadic view of the target.

Finally, we compared the weights used for the integration directly to the weights predicted by an optimal integration rule (Eq. 5). Particularly, we wanted to determine whether the weights are constant or whether they can be affected by changes in the underlying variances of the information sources. The predicted weights are based on the variability of the constituent target estimates derived from the remapping and static conditions. An increase in the post-saccadic target exposure time produced a decrease in the variability of the target estimate (Fig. 6A, Static). As a result, the predicted optimal weight associated with the postsaccadic target estimate increased with exposure time (Fig. $6 B$, dashed black line). Did the actual integration take into account this changing variability of the post-saccadic information and adjust its weight as a function of exposure time as predicted by optimal integration?

We used the average reaching direction in the combined condition (Fig. $7 B$ ) under post-saccadic target shifts to estimate the actual integration weights that were used by the subjects to combine the remapped and static target estimates (Eq. 4). These weights are plotted in Figure $6 B$ (dotted gray line). As predicted, the weight associated with the post-saccadic target was lowest when its variance was highest (at $75 \mathrm{~ms}$ exposure time). Indeed, the actual integration weight used by the participants showed a significant effect of exposure $\left(F_{(3,12)}=5.32 ; p=0.015\right)$. To compare the actual weights with the optimal weights, we performed a two-way, repeated-measures ANOVA with exposure and weight type as main effects. Only the effect of exposure time was significant $\left(F_{(3,12)}=6.16 ; p=0.009\right)$. Neither the weight-type main effect $\left(F_{(1,4)}=0.037 ; p=0.86\right)$ nor the interaction effect $\left(F_{(3,12)}=1.90 ; p=0.18\right)$ was found to be significant. Therefore, we observed a reasonably close match between the timedependent pattern of the actual integration weights and those predicted by optimal integration.

\section{Discussion}

When one fixates a reach target but then looks away, the brain produces an internal estimate of the remapped location of the reach target in fixation-centered coordinates (Batista et al., 1999; Medendorp et al., 2003; Crawford et al., 2004). A remapped internal estimate can be achieved by using a copy of the eye motor commands (Sommer and Wurtz, 2002) in conjunction with a forward model of the eye to predict where on the retina the image of the target should fall after the saccade. However, this remapping seems redundant because peripheral (static) vision of the reach target is available after the saccade and can be used to re-estimate its fixation-centered location. Indeed, reaches that are made to a target that has been remapped to peripheral vision produce errors that have a similar bias as reaches that are made to a static peripheral target (Henriques et al., 1998; Medendorp and Crawford, 2002). If both the remapped and peripherally perceived information is subject to the same bias, then what is the purpose of the remapped estimate?

One may argue that in a situation in which the reach target disappears, remapping is necessary to maintain a fixation- centered representation of the target. Alternatively, one may also suggest that the brain must have a way to differentiate between changes in visual input attributable to self-generated motion of the eyes compared with motion of the objects in the world. Our results provide an additional reason. In experiment 1 , we demonstrated that reaching to remapped targets produced errors that were equally biased as reaches made to static targets. However, reaches to remapped targets had significantly lower variance. In experiment 2, we found that the remapped target estimate was combined in an optimal manner with the post-saccadic target information to produce an overall target representation that was even more precise than the remapped estimate: the bias (combined-shift) and the variance (combined no-shift) of the final estimate of the target location were consistent with a Bayesian integration of the remapped and post-saccadic information about the target. Therefore, our results suggest that the brain generates an internal estimate of visual consequences of oculomotor commands, termed a forward model (von Holst, 1954; Miall et al., 1993), and combines this estimate with the actually observed visual feedback to produce an estimate of visual space that is far more accurate than is possible from either source alone.

Our results predict that this improved precision depends on whether the target is initially foveated. Foveal vision has high resolution, and the neural substrates involved in reaching toward foveal versus peripheral targets are distinct (Prado et al., 2005). Therefore, we predict that if a peripheral target is remapped, the remapped estimate will have higher uncertainty and its weight in a subsequent integration with actual sensory feedback will be lower than if the target was fixated initially.

In experiment 2, reach errors suggested that after a saccade, the remapped estimate of target location and the current visual feedback from the target were combined by weights that depended on their individual uncertainty. Such an estimate coincides with the Bayesian integration of the two information sources (assuming Gaussian distributions), in which the remapped estimate becomes a prior that is combined optimally with the new post-saccadic target information. Other studies provide support that the nervous system is capable of producing such Bayesian inference (Hinton et al., 1995; Ernst and Banks, 2002; Sahani and Dayan, 2003; Körding and Wolpert, 2004). An alternative explanation would be that the brain uses a constant weighting scheme independent of the uncertainty of the two information sources. We found a significant influence of exposure duration on the integration weights, arguing that the uncertainty of the information sources is used to determine the weights for integration. Although the change in weights matched the prediction under the assumption of optimal integration, the effect was rather small. Furthermore, we only varied the reliability of the post-saccadic, but not of the pre-saccadic, information. To test the hypothesis of optimal integration of visual information across saccades further, future studies might vary the uncertainty of both information sources over a larger range.

In describing the integration process, we assumed that the remapping and the subsequent static exposure to the target resulted in two independent representations that coexisted until they were integrated when the reach go-cue was given. This is reasonable because the remapped representation is memory based, whereas the static information is received in real time and so two distinct neural substrates might carry target information from the two sources. However, another possible integration mechanism consists of a single representation of the target that is continuously combined with new target information, using a Kalman filter. This means that the initial remapped representa- 
tion is integrated with post-saccadic information as soon as it is received and the resulting representation is again combined with the continuing post-saccadic information about the target.

One unresolved question is why both the peripherally perceived and remapped target estimates show a bias away from fixation. We replicated this finding, first reported by Henriques et al. (1998), in both of our experiments. It seems that although the forward model produced a less variable target estimate, it suffered from the same bias as a peripherally viewed target. Henriques et al. (1998) argued that the bias arose because the internal models for eye-hand coordination that account for the eye-head geometry were not calibrated under conditions of gaze deviation with the head unnaturally restrained (Henriques and Crawford, 2002). Following this idea, there would be no inherent biases in the target representations themselves (Poljac and Van Den Berg, 2003). Alternatively, the bias could arise in the perception of a peripheral target. The forward model that performs the remapping is constantly calibrated based on an error signal consisting of a difference between the predicted target estimate (remapped) and the biased sensory information (static condition). Initially, when remapping produces an unbiased target estimate, there would be a non-zero error signal that then adjusts the forward model to make its prediction similar to the biased target estimate of the static condition.

Another question that remains open is how awareness of the visual shift would influence the integration of pre-saccadic and post-saccadic information. We established in post interviews that our participants were not aware of the $2^{\circ}$ target shifts in the combined condition. This is the phenomenon of change blindness that refers to our inability to detect changes in stimulus position that occur during saccades [saccadic suppression (Mack, 1970; Bridgeman et al., 1975, 1979)] or when a blanking period precedes the reappearance of the stimulus (Deubel et al., 1996). Despite not being aware of the target shifts, participants displayed changes in reach direction consistent with the findings of Goodale et al. (1986). This lack of awareness is quite remarkable, given that the $\mathrm{SD}$ of the remapped and static target estimates was on the order of $0.44^{\circ}$ visual angle for remapping and $0.60^{\circ}$ for the static condition (accounted for control condition motor noise). Thus, the perceived difference between the pre-saccadic and post-saccadic target estimate would have a SD of 0.74 . This should have provided an optimal observer with enough information to detect a $2^{\circ}$ target jump quite reliably (correct on $99.6 \%$ of the trials; $\left.d^{\prime}=2.68\right)$. Nevertheless, participants integrated presaccadic and post-saccadic information believing that the target had remained stationary. In a pilot study, one participant became aware of the shifts, and he relied solely on the new post-saccadic information, as evidenced by his reach direction in the shifted combined condition. It would be valuable to systematically investigate how integration depends on the size and detectability of target shifts. Specifically, does visual awareness of the shift prevent the integration of the remapped prior? This remains to be explored.

Wolpert et al. (1995) made the seminal observation that during a reach, the brain estimated the state of the arm by integrating two sources of information: one derived from efferent copy and the other derived through proprioceptive feedback. Our results show that the same principle explains the estimate of the brain of visual reach targets following saccades and extends it by demonstrating that the neural mechanisms of estimation obey Bayesian rules of inference. Our proposed optimal integration of presaccadic and post-saccadic information regarding a reach target also relates to the idea that the perceptual representation of the environment is based on the integration of information across saccadic eye movements (Irwin, 1991; Niemeier et al., 2003; Brockmole and Irwin, 2005). Therefore, an important role of forward models is to provide an independent source of information about the sensory world, which is then combined statistically with actual sensations to form final estimates that guide our actions.

\section{References}

Ariff G, Donchin O, Nanayakkara T, Shadmehr R (2002) A real-time state predictor in motor control: study of saccadic eye movements during unseen reaching movements. J Neurosci 22:7721-7729.

Batista AP, Buneo CA, Snyder LH, Andersen RA (1999) Reach plans in eyecentered coordinates. Science 285:257-260.

Bock O (1986) Contribution of retinal versus extraretinal signals towards visual localization in goal-directed movements. Exp Brain Res 64:467-482.

Bridgeman B, Hendry D, Stark L (1975) Failure to detect displacement of visual world during saccadic eye movements. Vision Res 15:719-722.

Bridgeman B, Lewis S, Heit G, Nagle M (1979) Relation between cognitive and motor-oriented systems of visual position perception. J Exp Psychol Hum Percept Perform 5:692-700.

Brockmole JR, Irwin DE (2005) Eye movements and the integration of visual memory and visual perception. Percept Psychophys 67:495-512.

Crawford JD, Medendorp WP, Marotta JJ (2004) Spatial transformations for eye-hand coordination. J Neurophysiol 92:10-19.

Deubel H, Schneider WX, Bridgeman B (1996) Post-saccadic target blanking prevents saccadic suppression of image displacement. Vision Res 36:985-996.

Duhamel J-R, Colby CL, Goldberg ME (1992) The updating of the representation of visual space in parietal cortex by intended eye movements. Science 255:90-92.

Ernst MO, Banks MS (2002) Humans integrate visual and haptic information in a statistically optimal fashion. Nature 415:429-433.

Goodale MA, Pélisson D, Prablanc C (1986) Large adjustments in visually guided reaching do not depend on vision of the hand and perception of target displacement. Nature 320:748-750.

Henriques DY, Crawford JD (2002) Role of eye-head-shoulder geometry in the planning of accurate arm movements. J Neurophysiol 87:1677-1685.

Henriques DYP, Klier EM, Smith MA, Lowy D, Crawford JD (1998) Gazecentered remapping of remembered visual space in an open-loop pointing task. J Neurosci 18:1583-1594.

Hinton GE, Dayan P, Frey BJ, Neal RM (1995) The "wake-sleep" algorithm for unsupervised neural networks. Science 268:1158-1161.

Irwin DE (1991) Information integration across saccadic eye movements. Cogn Psychol 23:420-456.

Körding KP, Wolpert DM (2004) Bayesian integration in sensorimotor learning. Nature 427:244-247.

Mack A (1970) An investigation of the relationship between eye and retinal image movement in the perception of movement. Percept Psychophys 8:291-298.

Medendorp WP, Crawford JD (2002) Visuospatial updating of reaching targets in near and far space. NeuroReport 13:633-636.

Medendorp WP, Goltz HC, Vilis T, Crawford JD (2003) Gaze-centered updating of visual space in human parietal cortex. J Neurosci 23:6209-6214.

Merriam EP, Genovese CR, Colby CL (2003) Spatial updating in human parietal cortex. Neuron 39:361-373.

Miall RC, Weir DJ, Wolpert DM, Stein JF (1993) Is the cerebellum a Smith predictor? J Mot Behav 25:203-216.

Nakamura K, Colby CL (2002) Updating of the visual representation in monkey striate and extrastriate cortex during saccades. Proc Natl Acad Sci USA 99:4026-4031.

Nanayakkara T, Shadmehr R (2003) Saccade adaptation in response to altered arm dynamics. J Neurophysiol 90:4016-4021.

Niemeier M, Crawford JD, Tweed DB (2003) Optimal transsaccadic integration explains distorted spatial perception. Nature 422:76-80.

Poljac E, Van Den Berg AV (2003) Representation of heading direction in far and near head space. Exp Brain Res 151:501-513. 
Pouget A, Ducom JC, Torri J, Bavelier D (2002) Multisensory spatial representations in eye-centered coordinates for reaching. Cognition 83:1-11.

Prado J, Clavagnier S, Otzenberger H, Scheiber C, Kennedy H, Perenin MT (2005) Two cortical systems for reaching in central and peripheral vision. Neuron 48:849-858.

Sahani M, Dayan P (2003) Doubly distributional population codes: Simultaneous representation of uncertainty and multiplicity. Neural Comput 15:2255-2279.

Sommer MA, Wurtz RH (2002) A pathway in primate brain for internal monitoring of movements. Science 296:1480-1482.

Umeno MM, Goldberg ME (1997) Spatial processing in the monkey frontal eye field. I. Predictive visual responses. J Neurophysiol 78:1373-1383.

van Beers RJ, Sittig AC, Gon JJ (1999) Integration of proprioceptive and visual position information: an experimentally supported model. J Neurophysiol 81:1355-1364.

van Beers RJ, Haggard P, Wolpert DM (2004) The role of execution noise in movement variability. J Neurophysiol 91:1050-1063.

von Holst E (1954) Relations between the central nervous system and the peripheral organ. Br J Anim Behav 2:89-94.

Walker MF, Fitzgibbon J, Goldberg ME (1995) Neurons of the monkey superior colliculus predict the visual result of impeding saccadic eye movements. J Neurophysiol 73:1988-2003.

Wann JP, Ibrahim SF (1992) Does limb proprioception drift? Exp Brain Res 91:162-166.

Wolpert DM, Ghahramani Z, Jordan MI (1995) An internal model for sensorimotor integration. Science 269:1880-1882. 